\title{
The effect of palonosetron hydrochloride in the prevention of chemotherapy-induced moderate and severe nausea and vomiting
}

\author{
JIAN HUANG ${ }^{1}$, XIAO-JIA WANG ${ }^{1}$, DING YU ${ }^{2}$, YE-NING JIN ${ }^{3}$, LEI-ZHEN ZHEN ${ }^{4}$, \\ NONG XU ${ }^{5}$, WEI LIU ${ }^{6}$, YONG-CHUAN DENG ${ }^{7}$, SHI-XIU WU ${ }^{8}$ and JIA HE $^{9}$ \\ ${ }^{1}$ Zhejiang Cancer Hospital, Hangzhou 310022; ${ }^{2}$ Hubei Cancer Hospital, Wuhan 430079; ${ }^{3}$ Ruijin Hospital, Shanghai Jiao Tong \\ University School of Medicine, Shanghai 200025; ${ }^{4}$ Xinhua Hospital, Shanghai Jiao Tong University School of Medicine, \\ Shanghai 200092; ${ }^{5}$ The First Affiliated Hospital of College of Medicine, Zhejiang University, Hangzhou 310009; \\ ${ }^{6}$ Hebei Cancer Hospital, Shijiazhuang 050011; ${ }^{7}$ The Second Affiliated Hospital of College of Medicine, Zhejiang University, \\ Hangzhou 310008; ${ }^{8}$ The First Affiliated Hospital of Wenzhou Medical College, Hangzhou 325000; \\ ${ }^{9}$ Health Statistics Teaching and Research Section, The Second Army Medical University, \\ Shanghai 200433, P.R. China
}

Received November 21, 2012; Accepted January 14, 2013

DOI: $10.3892 / \mathrm{etm} .2013 .996$

\begin{abstract}
The current study aimed to evaluate the efficacy and safety of palonosetron hydrochloride injection for preventing chemotherapy-induced moderate and severe nausea and vomiting. A multi-centered, randomly stratified, doubleblind, double-dummy, parallel-group and positive-controlled trial was performed. A total of 240 patients who underwent chemotherapy treatment which induced moderate or severe vomiting were divided into the experimental and control groups. Half an hour before chemotherapy, the experimental group received a $0.25-\mathrm{mg}$ palonosetron hydrochloride injection, whereas the control group received a 3-mg granisetron injection. The acute vomiting complete remission rate (CRR) of the experimental group was not significantly different compared with that of the control group $(\mathrm{P}=0.35)$. The delayed vomiting CRR of the experimental group was significantly higher compared with that of the control group $(\mathrm{P}=0.002)$. No difference in full course vomiting CRR, vomiting control time, treatment failure time or acute nausea CRR was identified between the two groups. No significant differences in adverse events were observed between the experimental group and the control group. No significant differences in adverse reactions occurred between the experimental group and the control group (12.50\%). Palonosetron hydrochloride injection had a better effect on delayed vomiting CRR than granisetron hydrochloride injection. The two injections exhibited similar effects on acute vomiting CRR, full course vomiting CRR,
\end{abstract}

Correspondence to: Dr Xiao-Jia Wang, Zhejiang Cancer Hospital, No. 38 Guangji Road, Hangzhou, Zhejiang 310022, P.R. China E-mail: wxj88851@163.com

Key words: palonosetron, granisetron, chemotherapy, nausea, vomiting vomiting control time, treatment failure time (days), acute nausea CRR and adverse events.

\section{Introduction}

Chemotherapy is one of the main treatment methods for numerous types of cancer. However, it is capable of inducing the release of $5-\mathrm{HT}_{3}$ from enterochromaffin cells, which interacts with its receptors to cause vagal afferent nervous excitation, leading to the vomiting reflex. Nausea and vomiting are the two most common adverse reactions in cancer patients who receive chemotherapy. Inadequate control of these reactions often leads to a series of associated complications, which in turn may affect treatment outcome and compliance. Significant progress has been made in the prevention and treatment of chemotherapyinduced nausea and vomiting due to the application of 5- $\mathrm{HT}_{3}$ receptor antagonists. Commonly used first-generation $5-\mathrm{HT}_{3}$ receptor antagonists include ondansetron and granisetron. Although these drugs are able to achieve a complete control (CC) rate of 50-70\% for acute vomiting (1-5), they are not as effective in delayed vomiting, even in scenarios with repeated or combined medication plans (2). Therefore, the development of a more effective drug is urgently required.

Palonosetron hydrochloride injection belongs to the highly selective second generation of $5-\mathrm{HT}_{3}$ receptor antagonists. It was first developed by the the Helsin Healthcare S.A. Company (Lugano, Switzerland) and appeared on the market in the USA in July 2003 with the trade name Aloxi ${ }^{\mathrm{TM}}$ (6). However, whether this drug has a good curative effect or is safe for use among the Chinese population is unclear.

Therefore, a multi-centered clinical trial was conducted, with the support of the State Food and Drug Administration of China (no. 2007L00939) between September 2009 and September 2010. The drug involved in this study was generic palonosetron hydrochloride injection (a new drug produced by Zhejiang Puluo Kangyu Natural Medicine Co., Ltd., Jinhua, China). 


\section{Subjects and methods}

Subjects. Selection criteria for the subjects were as follows: i) They were histologically or cytologically diagnosed with malignant tumors (without tumor type restriction) and were required to receive chemotherapy; ii) no restrictions were imposed on their chemotherapy plan, which involved the application of chemotherapeutic drugs known to induce moderate or severe vomiting [the severity of chemotherapy-induced nausea was defined according to the NCCN Guidelines ${ }^{\circledR}$ for Antiemesis (8)]; iii) the patients did not receive any additional treatments, particularly chemotherapeutic drugs, from days 2-7 in a cycle of chemotherapy or antiemetics, tranquilizers, psychostimulants, antihistamines or hormones from days 1-7; iv) their age ranged from 18 to 75 years for either gender and their Karnofsky performance status scores were $\geq 60$; v) their life expectancy was $>3$ months; vi) their bone marrow was able to produce blood sufficiently; vii) they had normal liver and renal functions; viii) their electrocardiograms were basically normal, which satisfied chemotherapeutic eligibility; ix) they had recovered from toxic reactions (with the exception of baldness and nail changes) induced by previous treatment at least three weeks before the last time of radiotherapy and chemotherapy; and $\mathrm{x}$ ) they had signed an informed consent form. The study was approved by the ethics committee of Zhejiang Cancer Hospital, Hangzhou, China.

Study design. Since granisetron hydrochloride injection, which is currently administered in clinics, is apt to reduce nausea and vomiting, this study adopted a multi-centered, randomly stratified, double-blind, double-dummy, parallel-group and positive-controlled trial method. Based on stratification factors, including the severity of chemotherapy-induced nausea, gender and whether or not this was the first time the patient was receiving chemotherapy, the palonosetron hydrochloride injection (experimental) and granisetron hydrochloride injection (control) groups were created, with 120 patients allocated to each group. Eight centers were included in the study through competition and the central randomization system was applied.

Investigational drug. Palonosetron hydrochloride injection was provided by Zhejiang Puluo Kangyu Natural Medicine Co., Ltd. (batch no. 090504), whereas granisetron hydrochloride injection was provided by Ningbo Teampharm Co., Ltd. (batch no. 090301, Ningbo, China). A double-blind and double-dummy method was adopted. The experimental group were administered palonosetron hydrochloride injection $(0.25 \mathrm{mg})$ plus a granisetron hydrochloride injection simulated agent, whereas the control group were administered granisetron hydrochloride injection $(3 \mathrm{mg})$ plus a palonosetron hydrochloride injection stimulating agent. Randomization was performed using SAS software, in accordance with the stratification factors. An emergency letter for unblinding was prepared for each patient. The drugs were diluted with physiological saline to $40 \mathrm{ml}$ and administered to the patients half an hour before chemotherapy. The time taken to administer intravenous injection was $>5 \mathrm{~min}$.

Evaulation of curative effect. The curative effect for vomiting was evaluated according to the following criteria $(7,8)$ : i) Complete remission (CR), 0 times/24 h; ii) partial remission (PR), 1 time/24 h; iii) mild remission (MR), 2-5 times/24 h; and iv) failure $(\mathrm{F}),>5$ times/ $24 \mathrm{~h}$. The remission rates were calculated as follows: $\mathrm{CR}$ rate $=$ number of vomiting-free cases/total number of cases; PR rate $=$ number of $P R$ cases/total number of cases; and the effective rate = number of CR+PR+MR cases/ total number of cases. The three rate indices were calculated according to three observation intervals of acute (0-24 h), delayed (24-120 h) and full course (0-120 h) vomiting, respectively.

The effect on nausea was evaluated according to the following criteria: i) $\mathrm{CC}$, normal and nausea-free; and ii) partial control (PC), poor appetite with no changes in food habits (mild nausea) or decreased in food intake, no marked weight loss, dehydration or malnutrition and infusion time of $\leq 24 \mathrm{~h}$ (moderate nausea). The control rates were calculated as follows: $\mathrm{CC}$ rate $=$ number of $\mathrm{CC}$ cases/total number of cases and $\mathrm{PC}$ rate $=$ number of $\mathrm{CC}+\mathrm{PC}$ cases $/$ total number of cases.

Safety indices. The patients were observed every day during the trial and all adverse events were carefully recorded. Examination of vital signs, electrocardiograms, blood routine, urine routine, liver function, renal function and electrolyte levels were performed prior to and after treatment for safety. Adverse events were evaluated according to the National Cancer Institute CTCAE v3.0 criteria $(9,10)$.

Statistical analysis. Biostatistical analysis was performed by an independent third party (the Health Statistics Teaching and Research Section of The Second Army Medical University, Shanghai, China) using SAS 10.0 software. All rejected and suspended cases were statistically described. Demographic data and other basic indices were compared between the two groups using the $\chi^{2}$ test (or Fisher's exact probability test), Cochran-Mantel-Haenszel (CMH) test, Student's t-test and variance analysis or nonparametric statistical analysis methods. Test methods, including logistic regression analysis, the $\mathrm{CMH}$ test and covariance analysis, were carried out to the main curative indices for non-inferiority analysis and the non-inferiority threshold was determined to be $15 \%(\Delta=15 \%)$. Adverse events in the two groups were statistically described and compared using the $\chi^{2}$ test or the Fisher's exact probability test. Comparisons of vital signs, laboratory examination and electrocardiogram results were based upon the statistical description and analysis of changes observed prior to and after treatment. The mean values and incidence rates before and after drug administration were compared when necessary. $\alpha=0.05$ was used for all hypothesis tests.

\section{Results}

General data. A total of 240 patients were involved in this study and they were evenly divided into the experimental and control groups $(n=120)$. A total of 117 cases in the experimental group and 119 in the control group were enrolled in the full analysis set (FAS), 114 cases in the experimental group and 116 in the control group were enrolled in the per-protocol population set and 118 cases in the experimental group and 120 in the control group were enrolled in the safety analysis 
Table I. Comparison of basic data (count data/grade data) between two groups.

\begin{tabular}{|c|c|c|c|c|c|}
\hline Variable & Group A $(\%)$ & Group B (\%) & Test & Statistic & P-value \\
\hline \multicolumn{6}{|l|}{ Gender } \\
\hline Male & $31(26.50)$ & $32(26.89)$ & Exact test & & 1.000 \\
\hline Female & $86(73.50)$ & $87(73.11)$ & & & \\
\hline Total & 117 & 119 & & & \\
\hline \multicolumn{6}{|l|}{ Central nervous system involvement } \\
\hline No & $113(96.58)$ & $117(98.32)$ & Exact test & & 0.444 \\
\hline Yes & $4(3.42)$ & $2(1.68)$ & & & \\
\hline Total & 117 & 119 & & & \\
\hline \multicolumn{6}{|l|}{ Chemotherapy drugs } \\
\hline Moderately emetogenic chemotherapy & $22(18.80)$ & $21(17.65)$ & Exact test & & 0.867 \\
\hline Severely emetogenic chemotherapy & $95(81.20)$ & $98(82.35)$ & & & \\
\hline Total & 117 & 119 & & & \\
\hline \multicolumn{6}{|l|}{ Chemotherapy times } \\
\hline First & $61(52.14)$ & $58(48.74)$ & Exact test & & 0.606 \\
\hline Numerous times & $56(47.86)$ & $61(51.26)$ & & & \\
\hline Total & 117 & 119 & & & \\
\hline \multicolumn{6}{|l|}{ Other medical history } \\
\hline No & $87(74.36)$ & $86(72.27)$ & Exact test & & 0.769 \\
\hline Yes & $30(25.64)$ & $33(27.73)$ & & & \\
\hline Total & 117 & 119 & & & \\
\hline \multicolumn{6}{|l|}{ Drug combination } \\
\hline No & $60(51.28)$ & $51(42.86)$ & Exact test & & 0.240 \\
\hline Yes & $57(48.72)$ & $68(57.14)$ & & & \\
\hline Total & 117 & 119 & & & \\
\hline \multicolumn{6}{|l|}{ Tumor diagnosis } \\
\hline Non-small cell lung cancer & $27(23.08)$ & $23(19.33)$ & CMH test & 4.64 & 0.703 \\
\hline Colorectal cancer & $4(3.42)$ & $4(3.36)$ & & & \\
\hline Gastric cancer & $0(0.00)$ & $1(0.84)$ & & & \\
\hline Breast cancer & $71(60.68)$ & $73(61.34)$ & & & \\
\hline Esophageal cancer & $0(0.00)$ & $2(1.68)$ & & & \\
\hline Head and neck cancer & $2(1.71)$ & $1(0.84)$ & & & \\
\hline Ovarian cancer & $1(0.85)$ & $3(2.52)$ & & & \\
\hline Other & $12(10.26)$ & $12(10.08)$ & & & \\
\hline Total & 117 & 119 & & & \\
\hline \multicolumn{6}{|l|}{ Electrocardiogram } \\
\hline Normal & $72(61.54)$ & $67(56.30)$ & CMH test & 2.16 & 0.539 \\
\hline Abnormal, insignificance & $33(28.21)$ & $43(36.13)$ & & & \\
\hline Abnormal, significance & $8(6.84)$ & $5(4.20)$ & & & \\
\hline Unchecked & $4(3.42)$ & $4(3.36)$ & & & \\
\hline Total & 117 & 119 & & & \\
\hline
\end{tabular}

CMH test, Cochran-Mantel-Haenszel test. Group A, experimental group; Group B, control group.

data set. The number of suspended cases in the experimental and control groups were 1 and 0 and the number of rejected cases were 7 and 4 , respectively; no significant differences were identified. The demographic and baseline characteristics and vital signs of the two groups were similar and no significant differences were identified (Tables I and II).
Curative effect. No significant difference was identified between the acute vomiting CR rates of the experimental and control groups (49.12 vs. $42.24 \%$, respectively; $\mathrm{P}=0.350 ; 95 \%$ CI, -5.96-19.73; Table III). The non-inferiority test showed that palonosetron hydrochloride injection was not inferior to granisetron hydrochloride injection $(\mathrm{P}<0.001)$. 
Table II. Comparison of basic data (count data) between two groups.

\begin{tabular}{|c|c|c|c|c|c|}
\hline Variable & Group A & Group B & Test & Statistic & P-value \\
\hline \multicolumn{6}{|l|}{ Age (years) } \\
\hline $\mathrm{N}$ (missing) & $117(0)$ & $119(0)$ & Wilcoxon rank sum test & 0.06 & 0.951 \\
\hline Mean \pm SD & $52.06 \pm 10.46$ & $52.23 \pm 9.85$ & & & \\
\hline Median (Q1-Q3) & $53.00(44.00-60.00)$ & $53.00(46.00-60.00)$ & & & \\
\hline Min-Max & $31.00-72.00$ & $31.00-73.00$ & & & \\
\hline \multicolumn{6}{|l|}{ Height (cm) } \\
\hline $\mathrm{N}$ (missing) & $117(0)$ & $119(0)$ & Wilcoxon rank sum test & 0.54 & 0.586 \\
\hline Mean $\pm \mathrm{SD}$ & $161.68 \pm 7.22$ & $161.43 \pm 7.70$ & & & \\
\hline Median (Q1-Q3) & $160.00(156.00-168.00)$ & $160.00(156.00-168.00)$ & & & \\
\hline Min-Max & $147.00-180.00$ & $148.00-185.00$ & & & \\
\hline \multicolumn{6}{|l|}{ Weight (kg) } \\
\hline $\mathrm{N}$ (missing) & $117(0)$ & $119(0)$ & Group t-test & 0.60 & 0.546 \\
\hline Mean \pm SD & $59.78 \pm 9.19$ & $59.07 \pm 8.86$ & & & \\
\hline Median (Q1-Q3) & $59.00(54.00-65.00)$ & $58.50(53.00-65.00)$ & & & \\
\hline Min-Max & $42.00-85.00$ & $40.00-89.00$ & & & \\
\hline \multicolumn{6}{|l|}{ Temperature $\left({ }^{\circ} \mathrm{C}\right)$} \\
\hline $\mathrm{N}$ (missing) & $117(0)$ & $119(0)$ & Wilcoxon rank sum test & 0.51 & 0.610 \\
\hline Mean \pm SD & $36.77 \pm 0.38$ & $36.74 \pm 0.29$ & & & \\
\hline Median (Q1-Q3) & $36.80(36.50-37.00)$ & $36.80(36.50-37.00)$ & & & \\
\hline Min-Max & $36.00-38.30$ & $36.00-37.70$ & & & \\
\hline \multicolumn{6}{|l|}{ Heart rate (bpm) } \\
\hline $\mathrm{N}$ (missing) & $117(0)$ & $119(0)$ & Wilcoxon rank sum test & 0.74 & 0.459 \\
\hline Mean \pm SD & $79.10 \pm 4.84$ & $79.39 \pm 4.31$ & & & \\
\hline Median (Q1-Q3) & $80.00(78.00-82.00)$ & $80.00(78.00-80.00)$ & & & \\
\hline Min-Max & $62.00-92.00$ & $68.00-99.00$ & & & \\
\hline \multicolumn{6}{|l|}{ Breath } \\
\hline $\mathrm{N}$ (missing) & $117(0)$ & $119(0)$ & Wilcoxon rank sum test & 0.17 & 0.864 \\
\hline Mean \pm SD & $19.03 \pm 1.12$ & $19.08 \pm 1.18$ & & & \\
\hline Median (Q1-Q3) & $19.00(18.00-20.00)$ & $19.00(18.00-20.00)$ & & & \\
\hline Min-Max & $15.00-21.00$ & $17.00-22.00$ & & & \\
\hline \multicolumn{6}{|c|}{ Systolic pressure (mmHg) } \\
\hline $\mathrm{N}$ (missing) & $117(0)$ & $119(0)$ & Wilcoxon rank sum test & 1.36 & 0.172 \\
\hline Mean \pm SD & $120.33 \pm 14.69$ & $123.54 \pm 15.30$ & & & \\
\hline Median (Q1-Q3) & $120.00(110.00-130.00)$ & $120.00(110.00-133.00)$ & & & \\
\hline Min-Max & $91.00-164.00$ & $96.00-169.00$ & & & \\
\hline \multicolumn{6}{|c|}{ Diastolic pressure (mmHg) } \\
\hline $\mathrm{N}$ (missing) & $117(0)$ & $119(0)$ & Wilcoxon rank sum test & 0.12 & 0.906 \\
\hline Mean \pm SD & $76.73 \pm 8.32$ & $76.87 \pm 9.05$ & & & \\
\hline Median (Q1-Q3) & $78.00(70.00-80.00)$ & $77.00(70.00-82.00)$ & & & \\
\hline Min-Max & 56.00-100.00 & $60.00-114.00$ & & & \\
\hline \multicolumn{6}{|l|}{ KPS score } \\
\hline $\mathrm{N}$ (missing) & $117(0)$ & $119(0)$ & Wilcoxon rank sum test & 0.44 & 0.660 \\
\hline Mean \pm SD & $89.77 \pm 6.91$ & $89.17 \pm 7.66$ & & & \\
\hline Median (Q1-Q3) & $90.00(90.00-90.00)$ & $90.00(85.00-90.00)$ & & & \\
\hline Min-Max & 70.00-100.00 & 65.00-100.00 & & & \\
\hline \multicolumn{6}{|l|}{ Hemoglobin (g/l) } \\
\hline $\mathrm{N}$ (missing) & $117(0)$ & $119(0)$ & Wilcoxon rank sum test & 0.04 & 0.968 \\
\hline Mean \pm SD & $120.99 \pm 15.91$ & $120.70 \pm 15.79$ & & & \\
\hline Median (Q1-Q3) & $121.00(109.00-131.40)$ & $122.00(110.00-132.00)$ & & & \\
\hline Min-Max & $92.00-182.00$ & $90.00-169.00$ & & & \\
\hline
\end{tabular}


Table II. Continued.

\begin{tabular}{|c|c|c|c|c|c|}
\hline Variable & Group A & Group B & Test & Statistic & P-value \\
\hline \multicolumn{6}{|l|}{ White blood cell } \\
\hline $\mathrm{N}$ (missing) & $117(0)$ & $119(0)$ & Wilcoxon rank sum test & 0.12 & 0.907 \\
\hline Mean \pm SD & $6.65 \pm 2.83$ & $6.58 \pm 2.69$ & & & \\
\hline Median (Q1-Q3) & $6.20(5.00-7.40)$ & $5.90(5.10-7.40)$ & & & \\
\hline Min-Max & $3.00-22.40$ & $2.70-24.20$ & & & \\
\hline \multicolumn{6}{|l|}{ Neutrophil } \\
\hline $\mathrm{N}$ (missing) & $117(0)$ & $119(0)$ & Wilcoxon rank sum test & 0.12 & 0.903 \\
\hline Mean \pm SD & $4.50 \pm 2.52$ & $4.47 \pm 2.57$ & & & \\
\hline Median (Q1-Q3) & $3.84(3.00-5.20)$ & $3.90(2.90-5.30)$ & & & \\
\hline Min-Max & $1.80-18.80$ & $1.50-21.80$ & & & \\
\hline \multicolumn{6}{|l|}{ Platelet } \\
\hline $\mathrm{N}$ (missing) & $117(0)$ & $119(0)$ & Wilcoxon rank sum test & 0.52 & 0.603 \\
\hline Mean \pm SD & $251.25 \pm 91.56$ & $248.16 \pm 93.08$ & & & \\
\hline Median (Q1-Q3) & $241.00(183.00-303.00)$ & $235.00(180.00-298.00)$ & & & \\
\hline Min-Max & $71.00-741.00$ & $88.00-565.00$ & & & \\
\hline \multicolumn{6}{|c|}{ Total bilirubin $(\mu \mathrm{mmol} / \mathrm{l})$} \\
\hline $\mathrm{N}$ (missing) & $117(0)$ & $119(0)$ & Wilcoxon rank sum test & 1.78 & 0.075 \\
\hline Mean \pm SD & $9.04 \pm 4.32$ & $10.33 \pm 4.92$ & & & \\
\hline Median (Q1-Q3) & $8.50(6.50-12.00)$ & $9.56(6.80-13.20)$ & & & \\
\hline Min-Max & $0.16-24.30$ & $0.26-24.20$ & & & \\
\hline \multicolumn{6}{|c|}{ Direct bilirubin $(\mu \mathrm{mmol} / \mathrm{l})$} \\
\hline $\mathrm{N}$ (missing) & $117(0)$ & $119(0)$ & Wilcoxon rank sum test & 0.38 & 0.704 \\
\hline Mean \pm SD & $2.64 \pm 1.88$ & $2.69 \pm 1.86$ & & & \\
\hline Median (Q1-Q3) & $2.30(1.60-3.20)$ & $2.40(1.70-3.10)$ & & & \\
\hline Min-Max & $0.05-13.00$ & 0.06-14.90 & & & \\
\hline \multicolumn{6}{|l|}{ ALT (U/l) } \\
\hline $\mathrm{N}$ (missing) & $117(0)$ & $119(0)$ & Wilcoxon rank sum test & 0.30 & 0.766 \\
\hline Mean $\pm \mathrm{SD}$ & $25.63 \pm 19.31$ & $26.47 \pm 18.24$ & & & \\
\hline Median (Q1-Q3) & $20.00(14.20-30.00)$ & $21.00(14.80-32.40)$ & & & \\
\hline Min-Max & $7.00-141.00$ & 4.00-103.00 & & & \\
\hline \multicolumn{6}{|l|}{$\operatorname{AST}(\mathrm{U} / \mathrm{L})$} \\
\hline $\mathrm{N}$ (missing) & $117(0)$ & $119(0)$ & Wilcoxon rank sum test & 0.96 & 0.335 \\
\hline Mean \pm SD & $24.86 \pm 13.97$ & $24.42 \pm 12.99$ & & & \\
\hline Median (Q1-Q3) & $21.00(17.40-28.80)$ & $20.00(17.00-27.00)$ & & & \\
\hline Min-Max & $11.00-122.00$ & $11.00-77.00$ & & & \\
\hline \multicolumn{6}{|l|}{ Albumin (g/l) } \\
\hline $\mathrm{N}$ (missing) & $117(0)$ & $119(0)$ & Wilcoxon rank sum test & 0.02 & 0.985 \\
\hline Mean \pm SD & $40.35 \pm 5.21$ & $40.49 \pm 5.82$ & & & \\
\hline Median (Q1-Q3) & $40.50(37.00-44.10)$ & $40.30(37.00-43.90)$ & & & \\
\hline Min-Max & $23.00-50.90$ & $27.00-71.10$ & & & \\
\hline \multicolumn{6}{|l|}{ Glucose (mmol/l) } \\
\hline $\mathrm{N}$ (missing) & $109(8)$ & $116(3)$ & Wilcoxon rank sum test & 1.57 & 0.115 \\
\hline Mean \pm SD & $12.06 \pm 24.42$ & $8.65 \pm 16.03$ & & & \\
\hline Median (Q1-Q3) & $5.24(4.80-5.82)$ & $5.06(4.73-5.76)$ & & & \\
\hline Min-Max & 4.00-139.00 & $3.90-88.00$ & & & \\
\hline \multicolumn{6}{|l|}{$\mathrm{K}(\mathrm{mmol} / \mathrm{l})$} \\
\hline $\mathrm{N}$ (missing) & $112(5)$ & $114(5)$ & Wilcoxon rank sum test & 0.47 & 0.642 \\
\hline Mean $\pm \mathrm{SD}$ & $4.16 \pm 0.40$ & $4.13 \pm 0.50$ & & & \\
\hline Median (Q1-Q3) & $4.15(3.96-4.41)$ & $4.10(3.87-4.46)$ & & & \\
\hline Min-Max & $3.10-5.35$ & $1.44-5.66$ & & & \\
\hline
\end{tabular}


Table II. Continued.

\begin{tabular}{|c|c|c|c|c|c|}
\hline Variable & Group A & Group B & Test & Statistic & P-value \\
\hline \multicolumn{6}{|l|}{$\mathrm{Na}(\mathrm{mmol} / \mathrm{l})$} \\
\hline $\mathrm{N}$ (missing) & $112(5)$ & $114(5)$ & Wilcoxon rank sum test & 1.76 & 0.079 \\
\hline Mean $\pm \mathrm{SD}$ & $139.44 \pm 2.60$ & $140.01 \pm 2.71$ & & & \\
\hline Median (Q1-Q3) & $139.95(138.00-141.00)$ & $140.15(138.20-142.00)$ & & & \\
\hline Min-Max & $125.30-145.00$ & $132.10-147.40$ & & & \\
\hline \multicolumn{6}{|l|}{$\mathrm{Cl}(\mathrm{mmol} / \mathrm{l})$} \\
\hline $\mathrm{N}$ (missing) & $112(5)$ & $114(5)$ & Wilcoxon rank sum test & 1.79 & 0.074 \\
\hline Mean $\pm \mathrm{SD}$ & $102.47 \pm 2.92$ & $103.20 \pm 3.09$ & & & \\
\hline Median (Q1-Q3) & $102.90(101.00-104.05)$ & $103.25(101.70-105.00)$ & & & \\
\hline Min-Max & 89.90- 109.00 & 93.50- 112.80 & & & \\
\hline \multicolumn{6}{|l|}{ BUN (mmol/l) } \\
\hline $\mathrm{N}$ (missing) & $117(0)$ & $119(0)$ & Wilcoxon rank sum test & 1.51 & 0.130 \\
\hline Mean \pm SD & $4.95 \pm 2.35$ & $5.19 \pm 2.30$ & & & \\
\hline Median (Q1-Q3) & $4.33(3.68-5.18)$ & $4.63(3.93-5.90)$ & & & \\
\hline Min-Max & $1.50-18.10$ & 1.30-15.80 & & & \\
\hline \multicolumn{6}{|l|}{$\mathrm{Cr}(\mu \mathrm{mmol} / \mathrm{l})$} \\
\hline $\mathrm{N}$ (missing) & $117(0)$ & $119(0)$ & Wilcoxon rank sum test & 0.25 & 0.805 \\
\hline Mean \pm SD & $56.03 \pm 21.70$ & $57.58 \pm 23.33$ & & & \\
\hline Median (Q1-Q3) & $56.30(47.10-67.70)$ & $54.90(45.20-68.10)$ & & & \\
\hline Min-Max & $0.52-123.00$ & $0.55-183.00$ & & & \\
\hline
\end{tabular}

Group A, experimental group; Group B, control group;.ALT, glutamic-pyruvic transaminase enzyme; AST, glutamic-oxalacetic transaminease; BUN, urea nitrogen.

Table III. Comparison of the complete remission rate of acute vomiting between the two groups.

\begin{tabular}{lccc}
\hline Index & Experimental group (\%) & Control group (\%) & Statistics \\
\hline 0 grade & $56(49.12)$ & $49(42.24)$ & P-value \\
1 grade & $17(14.91)$ & $23(19.83)$ & $19(16.38)$ \\
2 grade & $23(20.18)$ & $25(21.55)$ & 0.89 \\
3 grade & $18(15.79)$ & 116 & 0.346 \\
Total & 114 & $49(42.24)$ & 0.87 \\
Efficacy & $56(49.12)$ & & 0.350 \\
Complete remission rate & a & & \\
\hline
\end{tabular}

${ }^{\mathrm{a} C}$ Complete remission rate 95\% confidence interval, -5.96-19.73.

A significant difference was identified between the delayed vomiting CR rates of the two groups (51.75 vs. $31.03 \%$; $\mathrm{P}=0.002$; 95\% CI, 8.27-33.17; Table IV). The non-inferiority test showed that palonosetron hydrochloride injection was not inferior to granisetron hydrochloride injection $(\mathrm{P}<0.001)$.

No significant difference was identified between the full course vomiting CR rates of the two groups (37.72 vs. $27.59 \%$; $\mathrm{P}=0.121 ; 95 \% \mathrm{CI},-1.92-22.19$; Table V). No significant difference was identified between the vomiting control times $(7.62 \pm 11.68$ vs. $9.72 \pm 16.01 ; \mathrm{P}=0.573$; Table VI), treatment failure times (days; $1.41 \pm 1.01$ vs. $1.36 \pm 0.70 ; \mathrm{P}=0.712$;
Table VII) or the acute vomiting CR rates (32.46 vs. $27.59 \%$; $\mathrm{P}=0.136$; Table VIII) of the two groups.

Safety. Adverse events in this study included any diseases, newly-emerged symptoms, abnormal vital signs or laboratory results and the aggravation of original symptoms or vital sign abnormalities occurring during the clinical drug trial, regardless of whether they were associated with the investigational drugs. Severe adverse events included death, threat to life, permanent or definite disabilities or handicaps, hospitalization or the extension of hospitalization length, congenital disabilities 
Table IV. Comparison of the complete remission rate of delayed vomiting between the two groups.

\begin{tabular}{lccr}
\hline Index & Experimental group (\%) & Control group (\%) & Statistics \\
\hline Complete remission & $59(51.75)$ & $36(31.03)$ & P-value \\
No complete remission & $55(48.25)$ & $80(68.97)$ & 116 \\
Total & 114 & $36(31.03)$ & 9.58 \\
Complete remission rate & $59(51.75)$ & 0.002 \\
\hline
\end{tabular}

${ }^{\mathrm{a} C}$ Complete remission rate 95\% confidence interval, 8.27-33.17.

Table V. Comparison of the complete remission rate of full vomiting between the two groups.

\begin{tabular}{lccr}
\hline Index & Experimental group (\%) & Control group (\%) & Statistics \\
\hline Complete remission & $43(37.72)$ & $32(27.59)$ & P-value \\
No complete remission & $71(62.28)$ & $84(72.41)$ & 116 \\
Total & 114 & $32(27.59)$ & 2.40 \\
Complete remission rate $^{\mathrm{a}}$ & $43(37.72)$ & 0.121 \\
\hline
\end{tabular}

${ }^{a}$ Complete remission rate $95 \%$ confidence interval, $-1.92-22.19$.

Table VI. Vomiting control time (hours) and comparison-FAS.

\begin{tabular}{|c|c|c|c|c|c|}
\hline Index & Experimental group & Control group & Test & Statistic & P-value \\
\hline N (missing) & $73(44)$ & $85(34)$ & Wilcoxon rank sum test & 0.56 & 0.573 \\
\hline Mean \pm SD & $7.62 \pm 11.28$ & $9.72 \pm 16.01$ & & & \\
\hline Median (Q1-Q3) & $3.00(0.00-8.00)$ & $3.70(0.50-12.00)$ & & & \\
\hline Min-Max & $0.00-50.00$ & $0.00-95.00$ & & & \\
\hline
\end{tabular}

FAS, full analysis set.

Table VII. Time to treatment failure (days) in the two groups and comparison-FAS.

\begin{tabular}{|c|c|c|c|c|c|}
\hline Index & Experimental group & Control group & Test & Statistic & P-value \\
\hline N (missing) & $22(95)$ & $33(86)$ & Wilcoxon rank sum test & 0.37 & 0.712 \\
\hline Mean \pm SD & $1.41 \pm 1.01$ & $1.36 \pm 0.70$ & & & \\
\hline Median (Q1-Q3) & $1.00(1.00-1.00)$ & $1.00(1.00-1.00)$ & & & \\
\hline Min-Max & $1.00-5.00$ & $1.00-3.00$ & & & \\
\hline
\end{tabular}

FAS, full analysis set.

Table VIII. Comparison of nausea complete response rate in the two groups; curative effect.

\begin{tabular}{lccc}
\hline Index & Experimental group $(\%)$ & Control group $(\%)$ & Statistics \\
\hline 0 grade & $37(32.46)$ & $32(27.59)$ \\
1 grade & $49(42.98)$ & $44(37.93)$ \\
2 grade & $25(21.93)$ & $35(30.17)$ \\
3 grade & $3(2.63)$ & $5(4.31)$ & 116 \\
Total & 114 & & 2.22 \\
Efficacy & & & 0.136 \\
\hline
\end{tabular}


Table IX. Occurrence of adverse event in the two groups.

\begin{tabular}{lrrrrrrrrr}
\hline & \multicolumn{3}{c}{ Experimental group } & & \multicolumn{3}{c}{ Control group } & \\
\cline { 2 - 3 } Events & Times & Cases & Incidence (\%) & & Times & Cases & Incidence (\%) & P-value \\
\hline Adverse events & 119 & 68 & 57.63 & & 145 & 81 & 67.50 & 0.141 \\
Important adverse events & 81 & 51 & 43.22 & & 90 & 51 & 42.50 & 1.000 \\
Serious adverse events & 0 & 0 & 0.00 & & 1 & 1 & 0.83 & 1.000 \\
Adverse reaction & 21 & 17 & 14.41 & & 18 & 15 & 12.50 & 0.707 \\
\hline
\end{tabular}

or birth defects, drug overdose and any other severe medical emergencies. Significant adverse events include those listed in adverse events and evident hematological or other laboratory test result abnormalities during drug administration, which may only be cured through targeted treatment.

In the experimental and control groups, $68(57.63 \%)$ and 81 cases $(67.50 \%)$, respectively, experienced adverse events, $51(43.22 \%)$ and 51 cases (42.50\%), respectively, experienced significant adverse events and $0(0.00 \%)$ and 1 case $(0.83 \%)$, respectively, experienced severe adverse events; no significant differences were identified between the two groups. Adverse reactions in the experimental and control groups were experienced in $17(14.41 \%)$ and 15 cases (12.50\%), respectively, and no significant difference was detected between the two groups (Table IX).

Adverse events which occurred at an incidence rate of $>10 \%$ included leucopenia $(11.02 \%$ in the experimental group and $22.50 \%$ in the control group) and myelosuppression ( $8.47 \%$ in the experimental group and $10.83 \%$ in the control group). These events were correlated with the bone marrow functionality of patients, as opposed to with the investigational drugs.

Grade 4 myelosuppression was the one severe adverse event which occurred in the control group, however this was not correlated with the investigational drug and was eventually cured.

Adverse reactions which were associated with the investigational drugs included constipation, asthenia, diarrhea, dizziness and abdominal distention. No statistically significant differences were observed between the two groups. The incidence rates of these reactions in either group were not $>10 \%$.

\section{Discussion}

The current study aimed to compare the efficacy and safety of palonosetron hydrochloride injection and granisetron hydrochloride injection in the prevention of chemotherapyinduced moderate and severe gastrointestinal reactions. It was a multi-centered, double-blind, double-dummy, parallelgroup, positive-controlled clinical trial. Chinese patients were administered palonosetron hydrochloride injection $(0.25 \mathrm{mg})$ or granisetron hydrochloride injection $(3 \mathrm{mg}$ ) half an hour before chemotherapeutic drug administration, for observation of the efficacy and safety of palonosetron hydrochloride injection in preventing chemotherapy-induced nausea and vomiting.
Between 22 September 2009 and 7 September 2010, a total of 240 patients from eight trial centers were investigated. They were divided into the experimental and control groups through central randomization according to certain factors, including gender, whether this was the first time the patient had received chemotherapy and the severity of chemotherapy-induced nausea. The demographic and baseline characteristics of the two groups were similar.

The results in this study demonstrated that there was no significant difference between the acute vomiting CR rates, full course vomiting $\mathrm{CR}$ rates, vomiting control times, treatment failure times, acute nausea CR rates or adverse events of the two groups, however, palonosetron hydrochloride injection did exhibit a better control on delayed vomiting compared with palonosetron hydrochloride injection. These results were identical to those previously reported (11), indicating that this drug has a good effect and high safety amongst the Chinese population.

Palonosetron has a 10-100 times higher affinity for $5-\mathrm{HT}_{3}$ receptors than other $5-\mathrm{HT}_{3}$ receptor antagonists (the pKi of palonosetron was 10.4 and those of granisetron, tropisetron and ondansetron are 8.91, 8.81 and 8.39, respectively) (12) and has an elimination half-life of $40 \mathrm{~h}$, which was markedly longer than that of other $5-\mathrm{HT}_{3}$ receptor antagonists (ondansetron, tropisetron and granisetron have half-lives of 4, 7.3 and $8.9 \mathrm{~h}$, respectively) (13-15). Therefore, palonosetron not only prevents chemotherapy-induced acute nausea and vomiting, but also has a positive preventative effect on delayed nausea and vomiting (16).

Since the single use of $5-\mathrm{HT}_{3}$ receptor antagonists is not able to completely control chemotherapy-induced nausea and vomiting, the option of an increase in the $5-\mathrm{HT}_{3}$ receptor antagonist dose or therapeutic combination with other drugs, for example glucocorticoids or $\mathrm{H}_{2}$ receptor antagonists, is often selected in clinical practice to enhance antiemetic effects (17-19).

Although antiemetic drugs with differing mechanisms of action, for example, the neurokinin-1 receptor blocker aprepitant (20), have already appeared on the worldwide market, $5-\mathrm{HT}_{3}$ receptor antagonists remain the major drugs used for antiemesis.

In conclusion, compared with granisetron hydrochloride injection, palonosetron hydrochloride injection has a better effect on delayed vomiting among the Chinese population. The two injections have a similar effect on the acute vomiting $\mathrm{CR}$ rate, full course vomiting $\mathrm{CR}$ rate, vomiting control time, 
treatment failure time (days), acute nausea CR rate and adverse event incidence rate of this population. Therefore, palonosetron hydrochloride injection has the potential to be widely administered in China.

\section{References}

1. Dempsey CL, Coop AJ, Shillington A, Farley PA, Eberhardt DR and O'Briant S: Antiemetic effectiveness of ondansetron and granisetron in patients with breast cancer treated with cyclophosphamide. Am J Health Syst Pharm 61: 781-786, 2004.

2. Geling $\mathrm{O}$ and Eichler HG: Should 5-hydroxytryptamine-3 receptor antagonists be administered beyond 24 hours after chemotherapy to prevent delayed emesis? Systematic re-evaluation of clinical evidence and drug cost implications. J Clin Oncol 23: 1289-1294, 2005.

3. Hesketh PJ: Comparative review of 5-HT3 receptor antagonists in the treatment of acute chemotherapy-induced nausea and vomiting. Cancer Invest 18: 163-173, 2000.

4. Jordan K, Hinke A, Grothey A, et al: A meta-analysis comparing the efficacy of four 5-HT3-receptor antagonists for acute chemotherapy-induced emesis. Support Care Cancer 15: 1023-1033, 2007.

5. Yonemura M, Katsumata N, Hashimoto H, et al: Randomized controlled study comparing two doses of intravenous granisetron ( 1 and $3 \mathrm{mg}$ ) for acute chemotherapy-induced nausea and vomiting in cancer patients: a non-inferiority trial. Jpn J Clin Oncol 39: 443-448, 2009.

6. Rubenstein EB: Palonosetron: a unique 5-HT3 receptor antagonist indicated for the prevention of acute and delayed chemotherapy-induced nausea and vomiting. Clin Adv Hematol Oncol 2: 284-289, 2004.

7. Kris MG, Gralla RJ, Clark RA, et al: Incidence, course and severity of delayed nausea and vomiting following the administration of high-dose cisplatin. J Clin Oncol 3: 1379-1384, 1985.

8. National Comprehensive Cancer Network: Antiemesis; V.2. 2009. http://www.nccn.org/professionals/physician_gls/PDF/ antiemesis.pdf. Accessed April 1, 2009.

9. National Cancer Institute: Common Terminology Criteria for Adverse Events v3.0 (CTCAE), 2006. http://ctep.cancer.gov/ protocolDevelopment/electronic_applications/docs/ctcaev3.pdf. Accessed August 9, 2006.

10. Roila F, Boschetti E, Tonato M, et al: Predictive factors of delayed emesis in cisplatin-treated patients and antiemetic activity and tolerability of metoclopramide or dexamethasone. A randomized single-blind study. Am J Clin Oncol 14: 238-242, 1991.
11. Balu S, Buchner D, Craver C and Gayle J: Palonosetron versus other 5-HT(3) receptor antagonists for prevention of chemotherapy-induced nausea and vomiting in patients with cancer on chemotherapy in a hospital outpatient setting. Clin Ther 33: 443-455, 2011

12. Ito H, Akuzawa $\mathrm{S}$, Tsutsumi R, et al: Comparative study of the affinities of the 5-HT3 receptor antagonists, YM060, YM114 (KAE-393), granisetron and ondansetron in rat vagus nerve and cerebral cortex. Neuropharmacology 34: 631-637, 1995.

13. Eglen RM, Lee CH, Smith WL, et al: Pharmacological characterization of RS 25259-197, a novel and selective 5-HT3 receptor antagonist, in vivo. Br J Pharmacol 114: 860-866, 1995.

14. Piraccini G, Stolz R, Tei M, et al: Pharmacokinetic Features of a Novel 5-HT3-Receptor Antagonist: Palonosetron (RS-25259-197). Proc Am Soc Clin Oncol 20: 400, 2001.

15. Wong EH, Clark R, Leung E, et al: The interaction of RS 25259-197, a potent and selective antagonist, with 5-HT3 receptors, in vitro. Br J Pharmacol 114: 851-859, 1995.

16. Botrel TE, Clark OA, Clark L, Paladini L, Faleiros E and Pegoretti B: Efficacy of palonosetron (PAL) compared to other serotonin inhibitors (5-HT3R) in preventing chemotherapy-induced nausea and vomiting (CINV) in patients receiving moderately or highly emetogenic (MoHE) treatment: systematic review and meta-analysis. Support Care Cancer 19: 823-832, 2011.

17. Campos D, Pereira JR, Reinhardt RR, et al: Prevention of cisplatin-induced emesis by the oral neurokinin-1 antagonist, MK-869, in combination with granisetron and dexamethasone or with dexamethasone alone. J Clin Oncol 19: 1759-1767, 2001.

18. de Wit R, de Boer AC, vd Linden GH, Stoter G, Sparreboom A and Verweij J: Effective cross-over to granisetron after failure to ondansetron, a randomized double blind study in patients failing ondansetron plus dexamethasone during the first 24 hours following highly emetogenic chemotherapy. Br J Cancer 85: 1099-1101, 2001.

19. Gralla RJ, de Wit R, Herrstedt J, et al: Antiemetic efficacy of the neurokinin-1 antagonist, aprepitant, plus a 5HT3 antagonist and a corticosteroid in patients receiving anthracyclines or cyclophosphamide in addition to high-dose cisplatin: analysis of combined data from two Phase III randomized clinical trials. Cancer 104: 864-868, 2005.

20. de Wit R, Herrstedt J, Rapoport B, et al: The oral NK(1) antagonist, aprepitant, given with standard antiemetics provides protection against nausea and vomiting over multiple cycles of cisplatin-based chemotherapy: a combined analysis of two randomised, placebo-controlled phase III clinical trials. Eur J Cancer 40: 403-410, 2004. 